\title{
New challenges in studying nutrition-disease interactions in the developing world
}

\author{
Andrew M. Prentice, ${ }^{1,2}$ M. Eric Gershwin, ${ }^{3}$ Ulrich E. Schaible, ${ }^{4}$ \\ Gerald T. Keusch, ${ }^{5}$ Cesar G. Victora, ${ }^{6}$ and Jeffrey I. Gordon 7
}

\begin{abstract}
${ }^{1}$ MRC International Nutrition Group, London School of Hygiene and Tropical Medicine, London, United Kingdom. ${ }^{2}$ MRC Keneba,
Keneba, The Gambia. ${ }^{3}$ Division of Rheumatology, Allergy and Clinical Immunology, Genome and Biomedical Sciences Facility, University of California, Davis, Davis, California, USA. ${ }^{4}$ Department of Infectious and Tropical Diseases, Immunology Unit, London School of Hygiene and Tropical Medicine, London, United Kingdom. 5Boston University Medical Campus and School of Public Health, Boston, Massachusetts, USA. ${ }^{6}$ Universidade Federal de Pelotas, Pelotas, Brazil. ${ }^{7}$ Center for Genome Sciences, Washington University School of Medicine, St. Louis, Missouri, USA.
\end{abstract}

\begin{abstract}
Latest estimates indicate that nutritional deficiencies account for 3 million child deaths each year in less-developed countries. Targeted nutritional interventions could therefore save millions of lives. However, such interventions require careful optimization to maximize benefit and avoid harm. Progress toward designing effective life-saving interventions is currently hampered by some serious gaps in our understanding of nutrient metabolism in humans. In this Personal Perspective, we highlight some of these gaps and make some proposals as to how improved research methods and technologies can be brought to bear on the problems of undernourished children in the developing world.
\end{abstract}

\section{Introduction}

Malnutrition, in its various guises, represents the greatest modifiable threat to global health and survival. This is especially so among children in the poorest nations of the world, where malnutrition and infections act hand in hand to create a self-reinforcing downward cycle of tissue depletion and lowered resistance to disease. Many of the solutions are already known and require political will, economic advancement, and operational research to achieve a resolution. In the interim, many international agencies are focusing attention on combating micronutrient deficiencies that lead to so-called "hidden hunger," since these are potentially amenable to short-term resolution. However, there remain a host of unsolved scientific questions that critically inhibit the development of such interventions that could potentially bring immediate health benefits and save millions of lives.

Space limitations preclude an exhaustive overview of the unknowns in the field. Instead, we present here a selective outline of some key research gaps, first emphasizing the global burden of childhood malnutrition. This discussion and a series of case studies of some unsolved nutritional issues serve as the foundation for proposing several challenges to the research community that, if overcome, we believe will lead to the development of interventions to combat nutrient deficiencies (see The challenges). We also hope that by posing here the challenges to overcoming a severe, but modifiable, problem of developing countries, we might help to create fresh momentum for the idea that advanced technologies and innovative approaches could help solve this age-old problem.

\section{The global burden of childhood malnutrition}

Childhood undernutrition is highly prevalent in low- and middleincome countries. Of all children born alive in such countries, $11 \%$

Nonstandard abbreviations used: APR, acute phase response; DALY, disabilityadjusted life year; TB, tuberculosis.

Conflict of interest: A.M. Prentice, U.E. Schaible, G.T. Keusch, and C.G. Victora have received research funds from the Bill and Melinda Gates Foundation. The remaining authors have declared that no conflict of interest exists.

Citation for this article: J. Clin. Invest. 118:1322-1329 (2008). doi:10.1172/JCI34034. have suffered from intrauterine growth restriction (1). Wasting (a measure of acute malnutrition detected as a low weight-for-height) affects $10 \%$ of these children, or about 55 million individuals (1). Stunting (a measure of chronic malnutrition detected as a low height-for-age) is an even larger problem, affecting $31 \%$ of children in low- and middle-income countries - approximately 170 million individuals worldwide (1). Being underweight (low weight-for-age) can result from stunting, wasting, or both and is used by the UN as an indicator of progress toward its first Millennium Development Goal: to eradicate extreme poverty and hunger by 2015 (http:// www.un.org/millenniumgoals/). Globally, the proportion of children who are underweight declined modestly from 33\% in 1990 to $27 \%$ in 2005 . However, slow progress in South Asia and sub-Saharan Africa forecasts that the first Millennium Development Goal will not be reached (2).

A recent analysis by the Maternal and Child Undernutrition Study Group (3) estimated that stunting, severe wasting, and intrauterine growth restriction together were responsible for 2.2 million deaths annually and $21 \%$ of disability-adjusted life years (DALYs), which are defined as the sum of the years of life lost due to premature mortality and the years of health and productivity lost due to disability for children younger than 5 years. Deficiencies of vitamin A and zinc were estimated to be responsible for 0.6 and 0.4 million deaths, respectively, and a combined $9 \%$ of global childhood DALYs. Iron and iodine deficiencies resulted in few child deaths, and combined were responsible for about $0.2 \%$ of global childhood DALYs. Iron deficiency as a risk factor for maternal mortality added 115,000 deaths and $0.4 \%$ of global total DALYs. Suboptimum breastfeeding was estimated to be responsible for 1.4 million child deaths each year and $10 \%$ of DALYs in children younger than 5 years. In an analysis that accounted for coexposure of these nutrition-related factors, they were together responsible for about $35 \%$ of child deaths and $11 \%$ of the total global disease burden (3). Undernourished children who survive to adulthood tend to have lower human capital, lower school achievement, reduced economic productivity, shorter adult height, and, for women, offspring with lower birth weight (4). To make things worse, children who suffered undernutrition in utero or in the first years of life, and who later gain weight rapidly - a common condi- 


\section{The challenges}

Challenge 1: To identify better and more comprehensive biomarkers of nutrient status in humans, as well as economic and accurate ways of measuring them.

Challenge 2: To expand the knowledge base with respect to host-pathogen competition for essential nutrients.

Challenge 3: To introduce additional rigor and sophistication into studies of the relationships between nutrient status and immune function.

Challenge 4: To emphasize large-scale prospective studies of nutritional contributors to infectious disease susceptibility, rather than post-hoc association studies.

Challenge 5: To characterize the significance of the human gut microbiome in modulating nutritional status, with a view to developing novel biomarkers of nutrient status and interventions based on manipulating the microbiota.

Challenge 6: To build the capacity to more rapidly translate basic discoveries related to the interrelationships between nutrient processing and metabolism, and variations in our human (and microbial) genomes, transcriptomes, proteomes, and metabolomes, into preventative and therapeutic interventions for children in the poorest nations of the world.

tion in middle-income and emerging countries - are at particular risk of chronic cardiovascular and metabolic diseases (5).

Every year, more than $\$ 70$ billion are spent on health research and development by the public and private sectors. An estimated $10 \%$ of this sum is used for research into $90 \%$ of the global burden of disease, leading to the so-called "10/90 gap" (6). In particular, the size of the research effort on nutritional topics is clearly insufficient to address a problem responsible for $10 \%-20 \%$ of all lost DALYs globally (Figure 1).

\section{The complexities of nutritional metabolism}

There are approximately 40 known essential nutrients. Each has generated a huge research literature, and when the potential interplay between nutrients is taken into consideration, the problem of defining their myriad effects on human physiology becomes apparent. In more recent years, attention has also focused on a large range of food-derived phytochemicals with diverse physiological roles whose health-protective effects have only recently started to emerge. These are just the biochemical complexities. There are also interactions between host genotype, diet, behavior, and social factors that ultimately govern diet-related disease patterns.

Zinc provides one example of some of the challenges involved in trying to understand diet-disease interactions. In humans, uptake of zinc from the diet and its subsequent distribution are regulated by a network of 24 known zinc transporter proteins (7). Zinc finger transcription factors play a key role in regulating our transcriptome, and knowledge of the fundamental role of zinc in gene replication and expression is advancing rapidly. Nonetheless, no precise mechanism has been identified by which zinc deficiency, or toxicity, might determine disease end-points of relevance to a child living in sub-Saharan Africa. Some randomized control trials in developing countries have shown that zinc supplementation can reduce morbidity and mortality resulting from diarrhea, pneumonia, and malaria and, in some groups, can enhance growth (8). However, recent very large randomized placebo control trials in Nepal and Pemba, each with over 40,000 children enrolled, reported no benefits of zinc supplementation on either morbidity (as assessed in the Nepal trial) or mortality (as assessed in both trials) $(9,10)$. Evidence about the effects of zinc supplementation is therefore equivocal, and because it is based on empirical studies there remain numerous questions, including: What is the mechanism(s) of action? What are the optimal dose levels, frequency, and mode? What is the optimal chemical form of administration? What are the appropriate age groups for treatment? And what diseases are amenable to such treatment? Moreover, claims that zinc deficiency plays a critical role in depressed immune function, altered cognitive development, and teratogenesis still have not been conclusively substantiated.

\section{Problems in assessing nutritional status}

The design of preventative nutritional interventions optimally requires a reliable assessment of the micronutrient deficiencies likely to be suffered in a given area or population. For certain nutrients there are well-defined, clinically detectable sequelae of deficiency (e.g., goiter for iodine deficiency, xerophthalmia for vitamin A deficiency, and anemia for iron deficiency). These sequelae can be used as crude indicators of geographic hot spots of deficiency in resident populations. However, they only detect the extremes of deficiency, representing the tip of the iceberg of the full health burden, and for many nutrients no such clinical indicators exist.

As an aid to understanding the nature and consequences of nutritional deficiencies, Golden proposed a novel system for classifying nutrients as Type I and Type II, according to the body's physiological responses to their deprivation (Figure 2) (11). In response to Type I nutrient deficiency, children's bodies continue to grow and use up the stored nutrient, eventually leading to tissue depletion, metabolic dysfunction, and consequent ill health. The illness has characteristic signs and symptoms, so the deficient nutrient can be identified and remedied. Examples of some Type I nutrients are iron, ascorbic acid, and vitamin A. With type II nutrient deficiency, the response is for the body to stop growing and repairing tissue, in order to conserve the nutrient, or even to break down its own tissues to make the nutrient available. Other Type II nutrients are lost in the process. No characteristic signs or symptoms differentiate any Type II nutrient deficiency from another, and detecting deficiency is complex. It is therefore almost impossible to determine which nutrient is causing the growth failure. Examples of Type II nutrients are potassium, magnesium, zinc, and protein.

\section{The conundrum of distinguishing cause from effect in studies of diet and disease}

Interactions between nutritional status and infectious diseases are bidirectional: certain nutrient deficiencies can promote susceptibility to infection and accelerate disease pathology, whereas infections lead to deficiencies through suppression of appetite 


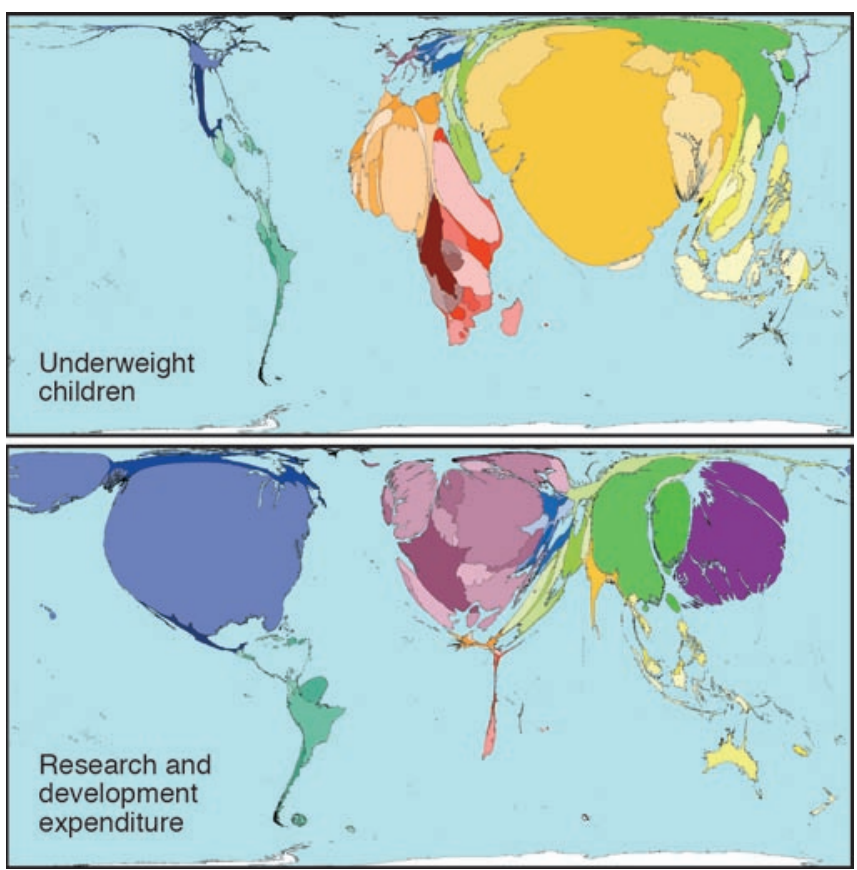

and cytokine-induced inhibition of nutrient absorption and tissue redistribution. The latter effects, characteristic of the acute phase response (APR), are generally assumed to be an active, evolutionarily selected part of the orchestrated host defense mechanisms designed to deprive microorganisms of nutritional substrate. For example, the APR results in massive changes in iron metabolism, with inhibition of intestinal absorption and iron release from hepatocytes and macrophages, resulting in a profound tissue redistribution. Systemic levels of zinc, selenium, retinol, riboflavin, pyridoxine, and ascorbate are also depressed by over $50 \%$, and copper levels rise (associated with a rise in ceruloplasmin). These changes greatly confound the interpretation of associations between nutritional status and disease outcomes.

To date, most studies in developing countries have assessed nutritional status in individuals with existing disease and are thus very difficult, if not impossible, to interpret. In countries of the developed world, this problem of establishing direction of causality has been properly addressed in numerous studies of chronic disease outcomes by using large population groups in whom dietary, lifestyle, and biochemical assessments made at baseline are

\section{Figure 2}

Differing pathophysiological responses to nutrient depletion. Nutritional deficiencies can be categorized as Type I or Type II according to the physiological responses of the body to dietary deficiency. In Type I deficiencies, growth continues in children, eventually resulting in tissues becoming depleted in the nutrient, leading to metabolic dysfunction and consequent ill health. As the illness has characteristic signs and symptoms, the deficient nutrient can be identified and remedied. With Type II nutrient deficiency, the response is for the body to stop growing and repairing tissue to conserve the nutrient, or even to break down its own tissues to make the nutrient available. Other Type II nutrients are lost in the process. No characteristic signs or symptoms differentiate one Type II nutrient deficiency from another, and detecting deficiency is complex. It is therefore almost impossible to determine which nutrient is causing the growth failure.

\section{Figure 1}

Inequity in science budgets in relation to the global burden of childhood undernutrition. Although more than $\$ 70$ billion are spent on health research and development by the public and private sectors each year, only an estimated $10 \%$ of this is used for research into $90 \%$ of the global burden of disease. For example, the amount of money spent on research into nutritional topics is far exceeded by the extent of the problem, which is responsible for $10 \%-20 \%$ of all lost DALYs globally. In these maps, countries are resized according to the prevalence of the indicator illustrated. Reproduced with permission from Worldmapper (http://www.worldmapper.org).

compared between subsequent disease cases and disease-free controls using a nested case-control design. There is an urgent need to apply such designs in less developed nations to identify the critical antecedent nutritional risk factors in relation to the major killer diseases: malaria, pneumonia, diarrheal illnesses, tuberculosis (TB), HIV, and life-threatening protein-energy malnutrition.

\section{The intersection between host nutrition and the immune system}

Undernourished children are profoundly more likely to die from a wide range of infections than their better-nourished counterparts (Figure 3) (12). Despite the widespread recognition of the implications of nutritional deficiencies on immune function, nearly all human studies have focused more on quantifying nutritional status than on dissecting the putatively associated immune responses. This reflects, in part, the difficulty of doing detailed functional immune assays in remote environments. For example, the literature contains innumerable studies of serum immunoglobulin levels and/or delayed hypersensitivity skin testing. In contrast, studies of the effects of nutritional deficiency on innate and adaptive immune responses to infectious agents are rare, and the possibility of nutritional supplementation improving responses to malaria, HIV, and TB remains elusive. We already know that the incidence of TB in the Western world decreased with improved nutrition and sanitation, but we do not know whether such improvement

Type I nutrient deficiency Child continues to grow Tissue levels of nutrient fall Specific symptoms arise

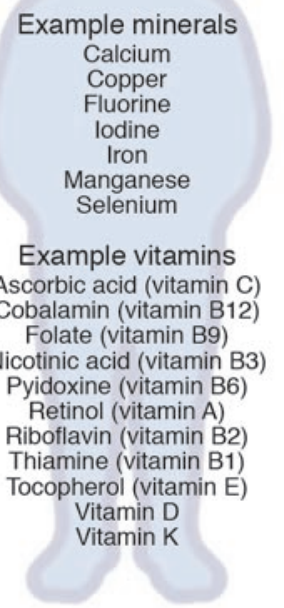

Type II nutrient deficiency Child growth slows Tissue levels of nutrient maintained No specific symptoms

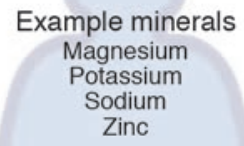

Example essential nutrients Water Energy Oxygen Nitrogen (protein) Phosphorus Sulfur Essential amino acids 


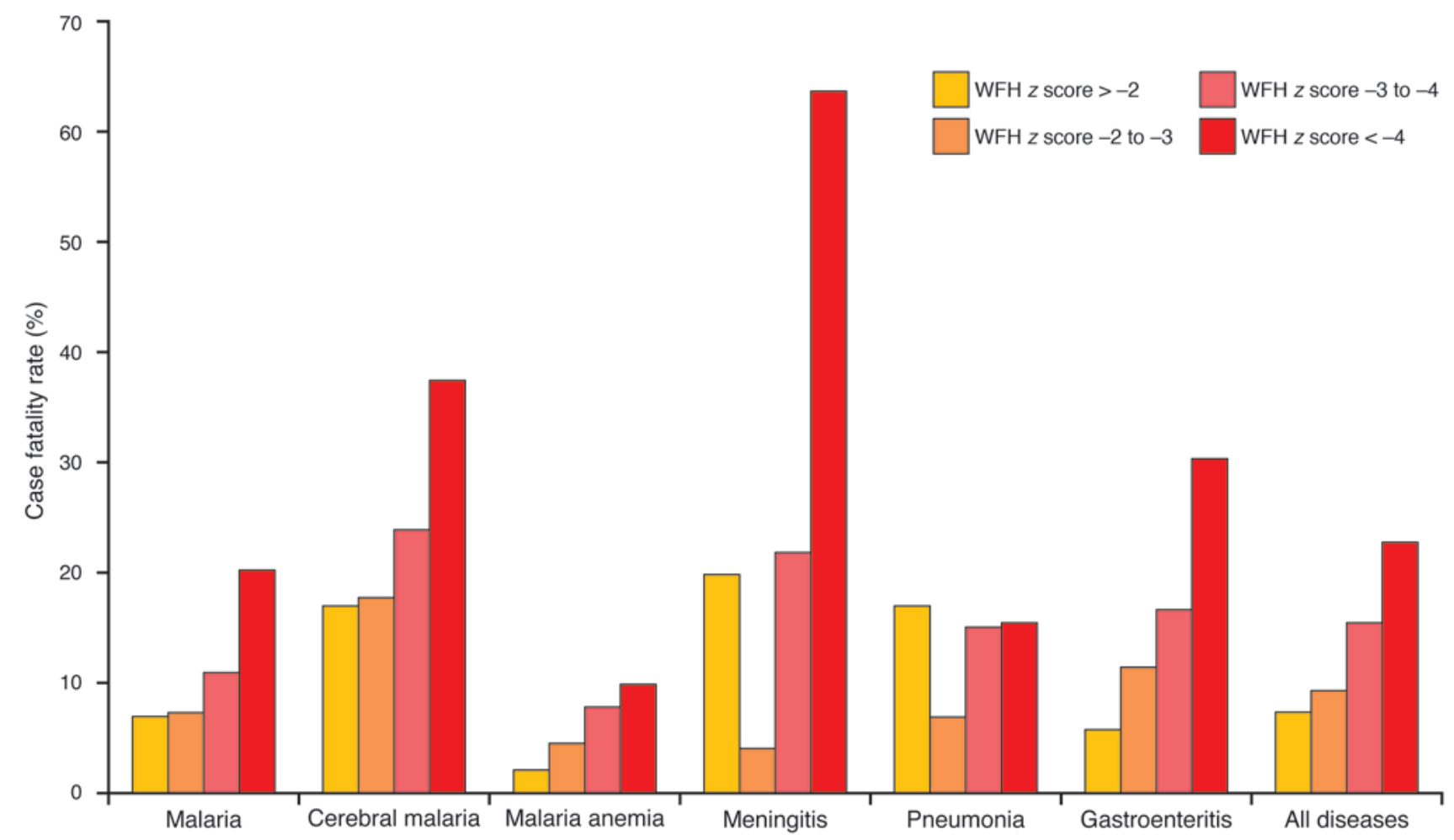

Figure 3

Influence of malnutrition on hospital case fatality rates for various diseases. Malnutrition is rarely identified as a specific cause of mortality, but it is an underlying contributory factor in at least one third of child deaths in developing countries. Depicted here are hospital case fatality rates from children admitted to Gambian hospitals with various primary diagnoses. The children analyzed were all malnourished, i.e., had low weight-for-height (WFH). As shown, the extent of malnutrition (WFH $z$ score $<-4$ being the most severely malnourished) correlates with the chance of an adverse survival outcome in most diseases. WFH $z$ score $<-4$, patients who are more than 4 standard deviations below the mean WFH; WFH $z$ score $=-3$ to -4 , patients who are 3-4 standard deviations below the mean WFH; WFH $z$ score $=-2$ to -3 , patients who are $2-3$ standard deviations below the mean WFH; WFH $z$ score $>-2$, patients who are less than 2 standard deviations below the mean WFH. Data collated by Man et al. (12).

was secondary to changes in transmission or to improved nutrition, and indeed, whether these might have been interlinked (see "Case study 3"). There are numerous other knowledge gaps. For example, we need to know the influence of nutritional status on recall (anamnestic) as well as primary immune responses. We need to define in a more vigorous and rigorous way the relationships between maternal nutrition, immunity, and transplacental transmission of immunoglobulins. We need to understand the biological mechanisms contributing to early-life nutritional programming of immune function and survival. We need to know whether nutritional improvement of mucosal immunity is possible. We need to know whether it is possible to modulate HIV transmission through nutritional means. And we need to know if we can augment the efficacy of future vaccines by accompanying nutritive measures.

\section{Host-pathogen competition for nutrients}

Micronutrient deficiencies enhance susceptibility to infection, so supplementation is frequently seen as beneficial in promoting resistance against infection. However, pathogenic microbes also require micronutrients for their growth. Therefore, nutritional interventions need critical evaluation to ensure that they do not benefit pathogens, thus causing disease exacerbation, activation of latent infections, and subsequently increased transmission rates (Figure 4).

Humans provide pathogens with habitats rich in essential nutrients. Consequently, a labile equilibrium is formed between host and pathogen (13). Mechanisms to sequester nutritive resources from pathogens are elementary parts of the mammalian host defense system, and in response, microbes have evolved diverse mechanisms for accessing nutrients from host sources. Although likely to be a rather universal phenomenon, details of host-pathogen competition are only known for a few micronutrients and a limited number of infections, and even for these exceptional cases, understanding is far from complete.

Probably the best-characterized trace element in this respect is iron, an essential factor for both host and pathogen (13). Microbes have high-affinity, multicomponent iron-uptake systems to compete with the host and inhabit intracellular habitats to access host iron reservoirs. Growth of many pathogenic bacteria, including species of Yersinia, Salmonella, Mycobacterium, Chlamydia, and Legionella, depends on host-derived iron (14). Consequently, iron overload in humans enhances susceptibility to these infections, as well as to malaria and AIDS, although in all cases the reasons for this effect are not well understood. This phenomenon has important implications for prophylactic iron supplementation. Clinically, anemia is considered a symptom requiring correction, and population-wide supplementation has been recommended based on some studies with beneficial outcome $(15,16)$. However, other studies revealed detrimental increases in the prevalence and severity of diarrhea, malaria, yersiniosis, salmonellosis, and TB $(17,18)$. Anemia can also be considered as an antimicrobial 


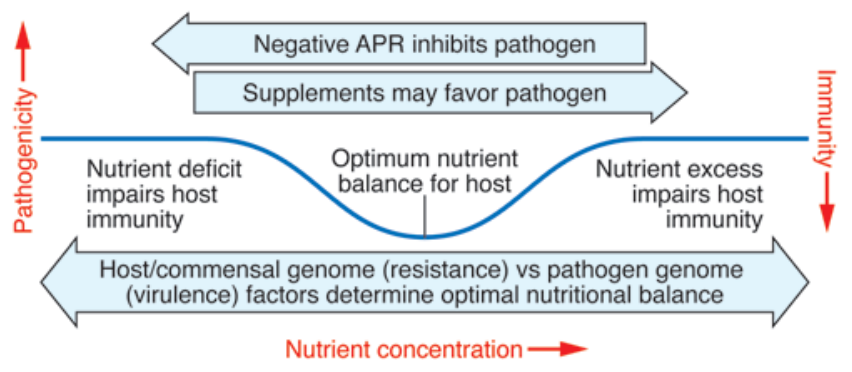

Figure 4

Optimizing nutritional status - a delicate and dynamic balance between the host and its pathogens. The optimal level for any individual's nutrient status is determined by a complex web of interacting parameters including their genetic background, environmental exposures, and interactions with other nutrients. In developing countries, and for certain nutrients (especially iron), host-pathogen competition for the nutrient adds an extra layer of complexity. Attempts to increase iron status conflict with the likelihood that excess iron might precipitate infections. The figure indicates that the optimum level (indicated by the nadir in the curve) varies according to host genotype (e.g., resistance factors involved in nutrient handling) and the genotypes of various pathogens (e.g., mechanisms of iron sequestration and consequent responses). By removing pathogen threats, as has largely been achieved in developed countries, it becomes possible to safely increase the optimum level of nutrient status with a view to improving cognitive and developmental outcomes.

response, and it has been proposed that a degree of anemia might represent an antiinfective adaptation (19). During inflammation, hepcidin, the hormone-like peptide and global regulator of iron homeostasis, causes anemia by decreasing intestinal iron uptake and promoting its sequestration inside macrophages (20). Other mechanisms also function to sequester iron from pathogens. For instance, lactoferrin helps to reduce biofilm formation by Psendomonas aeruginosa and controls growth of Mycobacterium tuberculosis in iron-overloaded hosts $(21,22)$. Lipocalin- 2 produced by epithelial cells and neutrophils binds bacterial-derived siderophores to recapture iron (23). IFN- $\gamma$ downregulates expression of the transferrin receptor to limit intracellular iron (24). In summary, iron metabolism and bioavailability are tightly regulated, and increasing the free iron pool might correct anemia but at the same time promote pathogen growth.

Pathogenic microbes often exploit the host for small organic molecules required for living, or as precursors for key biosynthetic pathways. There remains much to be learned about these interactions. Some microorganisms have lost certain biosynthetic genes, becoming auxotrophic and fully dependent on their (human) hosts. Chlamydia illustrates this point. It is sometimes a tryptophan auxotroph, relying on the host for this amino acid. As a host counter-strategy, IFN- $\gamma$ induces macrophages to express indoleamine 2,3-dioxygenase, which catabolizes L-tryptophan to $N$-formylkynurenine, hence limiting its bioavailability and driving Chlamydia into latency (25). Intriguingly, genetic variants in the tryptophan biosynthetic pathway determine habitat specificity and distinguish genital and ocular strains of Chlamydia (26). Arginine is a nonessential amino acid that serves as a precursor for reactive nitrogen intermediates produced by macrophages. Arginine has therefore been recommended as an "immunonutrient" (27). However, bacteria such as biofilm-forming Staphylococcus aureus, intracellular Liste- ria monocytogenes, and the intestinal parasite Giardia lamblia, all of which can directly consume arginine using efficient transport systems, might be able to take advantage of supplementary arginine $(28,29)$. Moreover, arginine-derived NO is consumed in microaerobic respiration by Neisseria meningitidis and P. aeruginosa strains using NO reductases $(30,31)$. Similar issues are likely to apply to many micronutrients including other amino acids, zinc, copper, fatty acids, and cholesterol.

In conclusion, a deeper understanding is needed of the molecular basis of micronutrient effects on the innate and adaptive immune systems of both model organisms and humans as well as their exploitability by pathogens. This information should form the foundation for hypothesis-based clinical trials that examine the impact of micronutrient/trace element supplementation on the host-pathogen relationship.

\section{Understanding the contributions of our microbiome to nutrition}

Most of our interactions with microbes are mutually beneficial, not pathogenic. We are born "germ-free" - a mixture of human somatic and germ cells. Beginning at birth, microbes are added to these starting materials. The process of colonization of our body surfaces is complex, dynamic, and driven by mechanisms that remain obscure. Nonetheless, recent work has emphasized how acquisition of our microbiota is likely to reflect a confluence of "legacy effects" (the microbes we encounter following birth), and body "habitat effects" (how human genotypes, immune systems, diets, and other factors define the chemical climate of those parts of our body that become home to our indigenous microbial communities) (32). By the time humans reach adulthood, microbial cells (primarily bacteria) outnumber human cells by as much as an order of magnitude. Most of these bacteria reside in the distal gut, where their density approaches one trillion organisms per milliliter of luminal contents. A few notable facts have emerged from 16S rRNA-based surveys of the distal gut microbiota of individuals living in Western societies (33-35). There are marked interpersonal differences in community composition at the species level. Although representatives of 10 of the 70 known bacterial divisions were identified in this body habitat, more than $90 \%$ of all phylogenetic types (phylotypes) belonged to members of just two of these divisions - the Bacteroidetes and the Firmicutes.

All of the genes embedded in the genomes of our microbial partners together comprise our microbiome and may exceed the number of genes in the Homo sapiens genome by several orders of magnitude (36). It follows that individual physiologic phenotypes need to be considered as a coevolved blend of human and microbial traits, and that understanding the underpinnings of human biologic diversity means that we need to make a commitment to characterizing the shared, as well as individual, features of our microbiomes. The Human Microbiome Project (HMP) is a response to this quest for self knowledge. Its launch coincided with a changing focus in the field of microbiology - from studying the properties and professions (niches) of single phylotypes under conditions that exist in the laboratory to studying the operations of whole communities in their native habitats. This more ecologic emphasis is now possible, in large part due to the introduction of a new generation of massively parallel DNA sequencers. The result is what has been called a new field of science, metagenomics (36). Metagenomics embraces cultiva- 
tion-independent, genome-anchored characterizations of intact microbial communities so that their assembly, composition, and operation can be defined at the level of their genomes, transcriptomes, proteomes, and metabolomes. Metagenomics should provide answers to a number of key questions such as, Do the interpersonal differences in human microbiota defined by $16 \mathrm{~S}$ rRNA gene sequencing accurately portray the degree of diversity in the microbiome? How are microbiomes shaped by host genotypes, environmental exposures, including exposure to our mothers and other family members, and lifestyles, including diet? And do interpersonal differences in gut microbiome structure and function affect predisposition to malnutrition?

\section{Some unsolved nutritional questions}

The above discussion highlights some of the many areas in which we believe that international nutrition research could benefit from the infusion of fresh ideas and experimental rigor. To illustrate these points, we describe four case studies that provide examples of past deficiencies and future opportunities.

Case study 1: Universal vitamin A supplementation - success, but with qualifications. The story of universal vitamin A supplementation for children provides some good lessons of how, even in one of nutrition's success stories, there could have been a more rigorous path to success, and there remain a number of serious controversies regarding mechanisms of action, optimal dosing regimens, and safety. Following the introduction of policies on worldwide use of iodized salt, the next micronutrient revolution involved vitamin A. In 1983, Sommer and colleagues reported the results of a longitudinal natural history study conducted in Indonesia, where they observed that preschool children with moderate vitamin A deficiency (Bitot's spots and night blindness) had an increased risk of death (37). This epidemiologic association led to a controlled clinical trial of vitamin A supplementation that was designed to determine whether periodic high-dose vitamin A supplementation could reduce child mortality (38). In 1987, the WHO and UNICEF issued a joint recommendation (39) for high-dose vitamin A treatment of all children with measles from communities in which vitamin A deficiency was a "recognized problem" or where the case fatality rates for measles were $1 \%$ or greater, because measles mortality was known to be strongly related to vitamin A status. In 1997, global advocacy by UNICEF encouraged community-based vitamin A supplementation in developing countries, and now most countries have adopted a national policy based on WHOrecommended dosing schedules (40).

Several lessons can be gleaned from this story. The proposal to promote vitamin A supplementation as an important intervention initially generated controversy because the effects observed seemed too good to be true, contradictory data were initially reported (41), there was no clearly demonstrable impact on the major causes of morbidity (42), and there was no plausible mechanism to explain a reduction in mortality (43). In addition, just one group of investigators had generated the supporting data from large field studies. It was not until other groups began to report similar results from different field settings, and the results of carefully conducted clinical trials were published supporting the effects of vitamin A, that supplementation was broadly accepted as a major public health intervention $(44,45)$. Moving forward to the present, we see that some of the impediments to moving from observation to intervention could perhaps have been more rapidly addressed by an integrated, inter- disciplinary basic and clinical research program that focused on how retinoids mediate host resistance to infection. Such an approach could have provided earlier insights into plausible mechanisms behind the large observed impact on mortality. This might have led to targeted interventions, in which specific retinoids with defined effects on host defense mechanisms might have been taken to clinical studies.

Although universal vitamin A supplementation is now accepted policy, the need for scientific clarity concerning its basic mode of action has never been greater. There is fresh controversy concerning optimal timing and dose levels, with recent suggestions that higher doses be given earlier in infancy (46) lacking support from experimental trials $(47,48)$. Moreover, there is evidence that vitamin A administration in the peripartum period has complex effects and, when taken daily, might actually enhance mother-to-child transmission of $\operatorname{HIV}(49,50)$. There are also suggestions that vitamin A might have negative interactions with some routine childhood vaccines, leading to excess later mortality, especially in girls (51).

Case study 2: Iron - more harm than good in some children? Recommendations for universal iron supplementation in areas with high rates of anemia, promoted for the best of motives by many international agencies, have also highlighted the depths of our collective ignorance about the benefits and possible harm of iron. Iron is the most commonly prescribed therapeutic agent, yet, as described above, it has potential toxic effects and may promote growth of pathogenic microbes. Can we safely combat anemia, and the other developmental deficits caused by iron deficiency, through universal iron supplementation programs? This question has been thrown into sharp relief by the premature termination by a data safety monitoring board of a very large trial of iron supplementation in children in Pemba Island after they detected a statistically significant increase in serious adverse events (hospitalization and deaths) in the groups receiving iron (18). Since Pemba has holoendemic malaria, and since a similar trial conducted in Nepal found no detrimental effect of iron, it has been concluded that the result arose from a negative interaction between iron and malaria. Earlier data would support this view, but it is by no means secure. Where do we go from here? A subsample analysis from Pemba suggested that the harmful effects were confined to children who were relatively iron replete, and that supplementation of the most deficient children would be safe.

The Pemba trial raises numerous questions at both the basic science and operational levels. What is the explanation of the adverse outcomes? Is it really because iron has promoted malaria-related morbidity? If so, does it promote malaria or malaria-related septicemia? Does this occur because supplementation involves nonphysiologic bolus doses of highly absorbable iron, overwhelming the children's iron-handling apparatus and resulting in non-transferrin-bound iron (NTBI) that is available to resident pathogens? If so, can we design better formulations for the safer delivery of iron? At the operational level, if we need to screen populations in order to target supplementation, we need new portable and reliable methods for assessing iron status.

Case study 3: Nutrition and TB - more unknown than known. TB is one of the most important human infectious diseases, with 1.7 million deaths and 8 million new cases annually. TB is generally accepted as being closely linked to nutritional status, yet there is a lamentable lack of a solid evidence base on which to incor- 
porate nutritional interventions into clinical practice or community preventative strategies. Several reports have suggested protein energy malnutrition as a risk factor (52-54). Weight loss, cachexia, and anemia caused by inflammatory responses to the infection contribute further to the poor nutritional status of patients with TB. Serum zinc concentrations are below those of healthy controls, whereas copper concentrations are higher $(55,56)$. However, these changes are characteristic of the APR, and the studies fall foul of the reverse causality trap that can only be circumvented by prospective nested case-control studies. Recent work has also revealed a potentially important connection between vitamin $\mathrm{D}$ and innate immunity in individuals with TB. Vitamin D promotes expression of the anti-mycobacterial peptide, cathelicidin (57). Therefore, lower serum concentrations of vitamin D might contribute to diminished immunity against TB and provide a target for prophylactic as well as therapeutic correction by dietary vitamin D supplementation. Beyond these few glimpses into the likely influence of nutrition on susceptibility to TB and disease progression, it is difficult to find any other authoritative information.

Case study 4: Nutritional modulation of pathogenicity. Our final case study illustrates how host nutritional status can affect the basic biology of viruses. Beck and colleagues have demonstrated that host deficiencies in the antioxidant nutrients selenium and vitamin E allow mildly pathogenic strains of coxsackie viruses and influenza viruses to mutate into virulent strains (reviewed in ref. 58). The enhanced pathogenicity is genomically encoded and hence maintained when passaged through nutrient-replete animals. These findings raise numerous related questions. With many pandemic viruses (e.g., HIV, influenza, Avian flu) originating in the poorest regions of developing countries, the question arises as to whether nutrient deficiencies have created conditions for the origination of novel pathogenic strains. There is an urgent need to assess these processes in humans and to extend the original work on viral mutations to other microorganisms. Emergence of exceptionally virulent drug-resistant strains of TB are assumed to be driven by a combination of poorly implemented drug regimens and coinfection with HIV, which lowers immunosurveillance of emerging strains, but host malnutrition might play a contributory role and merits investigation.

\section{The way forward}

The usual arguments used to persuade leading scientists to engage with issues affecting the developing world revolve around the philanthropic principle. This also drives the strategic deployment of funds by many private, governmental, and international agencies that are committed to sponsoring scientific programs designed to address global health issues. We contend that as global disease patterns increasingly merge, and because the basic science underpinning many nutrient-disease interactions can best be studied in "deplete" populations, there is an additional motive of self-interest in engaging with the problems of the poorest peoples of the world.

The more fully we understand the mechanisms linking diet, health, and disease, the more effective will be our ability to design optimal nutrient interventions. At this time of remarkable and explosive expansion in our ability to characterize our individual human genomes, transcriptomes, proteomes, and metabolomes as well as the impact of our environmental exposures and rapidly changing lifestyles on their operations, we cannot justify empirical guesses as to which nutrient interventions might benefit underprivileged children and adults whose lives are in jeopardy. The individual challenges set out in The challenges represent only a few among many areas in which international nutrition science could be strengthened. A theme linking these challenges is that they require a deeper incorporation of the concepts and experimental techniques of multiple scientific disciplines into the field of nutrition. We hope that this brief and self-critical survey of our field will stimulate interaction with investigators outside our field who are supremely qualified to contribute these techniques and expertise to studies of human nutrition.

\section{Acknowledgments}

We thank Ellen Piwoz and Fil Randazzo from the Bill and Melinda Gates Foundation for their valuable comments.

Address correspondence to: Andrew Prentice, MRC International Nutrition Group, London School of Hygiene and Tropical Medicine, Keppel Street, London WC1E 7HT, United Kingdom. Phone: 44-20-7958-8140; Fax: 44-20-7958-8111; E-mail: andrew.prentice@ lshtm.ac.uk.
1. UNICEF. 2006. The State of the World's Children 2007: executive summary. http://www.unicef.org/ publications/index_36602.html.

2. United Nations. 2007. The Millennium Development Goals Report 2007. http://www.un.org/millenniumgoals/pdf/mdg2007.pdf.

3. Black, R.E., et al. 2008. Maternal and child undernutrition: global and regional exposures and health consequences. Lancet. 371:243-260.

4. The World Bank. 2006. Repositioning nutrition as central to development: a strategy for large-scale action. The World Bank. Washington, DC, USA. 246 pP.

5. Prentice, A.M., and Moore, S.E. 2005. Early programming of adult diseases in resource poor countries. Arch. Dis. Child. 90:429-432.

6. Global Forum for Health Research. 2004. 10/90 report on health research 2003-2004. http://www.globalforumhealth.org/Site/002 What $\% 20$ we\%20do/005__Publications/001 10\%2090\%20reports.php.

7. Liuzzi, J.P., and Cousins, R.J. 2004. Mammalian zinc transporters. Annu. Rev. Nutr. 24:151-172.

8. Black, R.E. 2003. Zinc deficiency, infectious disease and mortality in the developing world. J. Nutr. 133:1485S-1489S
9. Tielsch, J.M., et al. 2007. Effect of daily zinc supplementation on child mortality in southern Nepal: a community-based, cluster randomised, placebocontrolled trial. Lancet. 370:1230-1239.

10. Sazawal, S., et al. 2007. Effect of zinc supplementation on mortality in children aged 1-48 months: a community-based randomised placebo-controlled trial. Lancet. 369:927-934.

11. Golden, M.H. 1995. Specific deficiencies versus growth failure: type I and type II nutrients. SCN News. 12:10-14.

12. Man, W.D., et al. 1998. Nutritional status of children admitted to hospital with different diseases and its relationship to outcome in The Gambia, West Africa. Trop. Med. Int. Health. 3:678-86.

13. Schaible, U.E., and Kaufmann S.H. 2005. A nutritive view on the host-pathogen interplay. Trends Microbiol. 13:373-380.

14. Schaible, U.E., and Kaufmann S.H. 2004. Iron and microbial infection. Nat. Rev. Microbiol. 2:946-953.

15. Iannotti, L.L., Tielsch, J.M. Black, M.M., and Black, R.E. 2006. Iron supplementation in early childhood: health benefits and risks. Am. J. Clin. Nutr. 84:1261-1276

16. Tielsch, J.M., et al. 2006. Effect of routine prophy- lactic supplementation with iron and folic acid on preschool child mortality in southern Nepal: community-based, cluster-randomised, placebocontrolled trial. Lancet. 367:144-152.

17. Murray, M.J., Murray, A.B., Murray, M.B., and Murray, C.J. 1978. The adverse effect of iron repletion on the course of certain infections. Br. Med.J. 2:1113-1115.

18. Sazawal, S., et al. 2006. Effects of routine prophylactic supplementation with iron and folic acid on admission to hospital and mortality in preschool children in a high malaria transmission setting: community-based, randomised, placebo-controlled trial. Lancet. 367:133-143.

19. Denic, S., and Agarwal, M.M. 2007. Nutritional iron deficiency: an evolutionary perspective. Nutrition. 23:603-614.

20. Weiss, G., and Goodnough, L.T. 2005. Anemia of chronic disease. N. Engl. J. Med. 352:1011-1023.

21. Singh, P.K., Parsek, M.R., Greenberg, E.P., and Welsh, M.J. 2002. A component of innate immunity prevents bacterial biofilm development. Nature. 417:552-555.

22. Schaible, U.E., Collins, H.L., Priem, F., and Kaufmann, S.H. 2002. Correction of the iron over- 
load defect in beta-2-microglobulin knockout mice by lactoferrin abolishes their increased susceptibility to tuberculosis. J. Exp. Med. 196:1507-1513.

23. Borregaard, N., and Cowland, J.B. 2006. Neutrophil gelatinase-associated lipocalin, a siderophore-binding eukaryotic protein. Biometals. 19:211-215.

24. Byrd, T.F., and Horwitz, M.A. 1989. Interferon gamma-activated human monocytes downregulate transferrin receptors and inhibit the intracellular multiplication of Legionella pneumophila by limiting the availability of iron. J. Clin. Invest. 83:1457-1465.

25. MacKenzie, C.R., Heseler, K., Muller, A., and Daubener, W. 2007. Role of indoleamine 2, 3-dioxygenase in antimicrobial defence and immuno-regulation: tryptophan depletion versus production of toxic kynurenines. Curr. Drug Metab. 8:237-244.

26. Caldwell, H.D., et al. 2003. Polymorphisms in Chlamydia trachomatis tryptophan synthase genes differentiate between genital and ocular isolates. J. Clin. Invest. 111:1757-1769.

27. Wilmore, D. 2004. Enteral and parenteral arginine supplementation to improve medical outcomes in hospitalized patients. J. Nutr. 134:2863S-2867S.

28. Zhu, Y., et al. 2007. Staphylococcus aureus metabolism in a biofilm: the influence of arginine on polysaccharide intercellular adhesin synthesis, biofilm formation, and pathogenesis. Infect. Immun. 75:4219-4226.

29. Eckmann, L., et al. 2000. Nitric oxide production by human intestinal epithelial cells and competition for arginine as potential determinants of host defense against the lumen-dwelling pathogen Giardia lamblia. J. Immunol. 164:1478-1487.

30. Stevanin, T.M., Moir, J.W., and Read, R.C. 2005 Nitric oxide detoxification systems enhance survival of Neisseria meningitidis in human macrophages and in nasopharyngeal mucosa. Infect. Immun. 73:3322-3329.

31. Kakishima, K., Shiratsuchi, A., Taoka, A., Nakanishi, Y., and Fukumori, Y. 2007. Participation of nitric oxide reductase in survival of Pseudomonas aeruginosa in LPS-activated macrophages. Biochem. Biophys. Res. Commun. 355:587-591.

32. Rawls, J.F., Mahowald, M.A., Ley, R.E., and Gordon, J.I. 2006. Reciprocal gut microbiota transplants from zebrafish and mice to germ-free recipients reveal host habitat selection. Cell. 127:423-433.

33. Eckburg, P.B., et al. 2005. Diversity of the human intestinal microbial flora. Science. 308:1635-1638.

34. Ley, R.E., Turnbaugh, P.J., Klein, S., and Gordon, J.I. 2006 Human gut microbes linked to obesity. Nature. 444:1022-1023.

35. Ley, R., Peterson, D.A., and Gordon, J.I. 2006 Ecological and evolutionary forces that shape microbial diversity and genome content in the human intestine. Cell. 124:837-848.

36. Committee on Metagenomics. 2007. The new science of metagenomics: revealing the secrets of our microbial planet. National Academies Press. Washington, DC, USA. 170 pP.

37. Sommer, A., Tarwotjo, I., Hussaini, G., and Susanto, D. 1983. Increased mortality in children with mild vitamin A deficiency. Lancet. 2:585-588.

38. Sommer, A., et al. 1986. Impact of vitamin A supplementation on childhood mortality. A randomized controlled community trial. Lancet. 8491:1169-1173.

39. WHO and UNICEF. 1987. Joint WHO/UNICEF statement on vitamin A for measles. Weekly Epidemiological Record. 62:133-134.

40. WHO. 1995. Vitamin A deficiency and its consequences: a filed guide to their detection and control. 3rd edition. Sommer, A., editor. WHO. Geneva, Switzerland. $93 \mathrm{pp}$.

41. Herrera, M.G., et al. 1992. Vitamin A supplementation and child survival. Lancet. 340:267-271.

42. Rahmathullah, L., Underwood, B.A., Thulasiraj, R.D., and Milton, R.C. 1991. Diarrhea, respiratory infections, and growth are not affected by a weekly low-dose vitamin A supplement: a masked, controlled field trial in children in southern India. Am. J. Clin. Nutr. 54:568-577.

43. Abdeljaber, M.H., Monto, A.S., Tilden, R.L., Schork, M.A., and Tarwotjo, I. 1993. The impact of vitamin A supplementation on morbidity: a randomized community intervention. Am. J. Public Health. 81:1654-1656.

44. Fawzi, W.W., Chalmers, T.C., Herrera, M.G., and Mosteller, F. 1993. Vitamin A supplementation and child mortality. A meta-analysis. JAMA. 269:898-903

45. Keusch, G.T. 1990. Vitamin A supplements-too good not to be true. N. Engl. J. Med. 323:985-987.
46. Sommer, A., and Davidson, F.R. 2002. Assessment and control of vitamin A deficiency: the Annecy Accords. J. Nutr. 132(9 Suppl.):2845S-2850S

47. Darboe, M.K., et al. 2007. Effectiveness of an early supplementation scheme of high-dose vitamin A versus standard WHO protocol in Gambian mothers and infants: a randomised controlled trial. Lancet. 369:2088-2096

48. Benn, C.S., et al. 2005. Randomised study of effect of different doses of vitamin A on childhood morbidity and mortality. BMJ. 331:1428-1432.

49. Fawzi, W.W., et al. 2002. Randomized trial of vitamin supplements in relation to transmission of HIV-1 through breastfeeding and early child mortality. AIDS. 16:1935-1944.

50. Humphrey, J.H., et al. 2006. Effects of a single large dose of vitamin A, given during the postpartum period to HIV-positive women and their infants, on child HIV infection, HIV-free survival, and mortality. J. Infect. Dis. 193:860-871.

51. Benn, C.S., Fisker, A.B., Jorgensen, M.J., and Aaby, P. 2007. Why worry: vitamin A with DTP vaccine? Vaccine. 25:777-779.

52. Schaible, U.E., and Kaufmann, S.H. 2007. Malnutrition and infection: complex mechanisms and global impacts. PLoS Med. 4:e115.

53. Cegielski, J.P., and McMurray, D.N. 2004. The relationship between malnutrition and tuberculosis: evidence from studies in humans and experimental animals. Int. J. Tuberc. Lung Dis. 8:286-298.

54. van Lettow, M., Fawzi, W.W., and Semba, R.D. 2003. Triple trouble: the role of malnutrition in tuberculosis and human immunodeficiency virus co-infection. Nutr. Rev. 61:81-90.

55. van Lettow, M., et al. 2004. Micronutrient malnutrition and wasting in adults with pulmonary tuberculosis with and without HIV co-infection in Malawi. BMC Infect. Dis. 4:61

56. Bogden, J.D., Lintz, D.I., Joselow, M.M., Charles, J. and Salaki, J.S. 1978. Copper/zinc ratios in whole blood, plasma, and erythrocytes in pulmonary tuberculosis. Health Lab. Sci. 15:38-43.

57. Liu, P.T., et al. 2006. Toll-like receptor triggering of a vitamin D-mediated human antimicrobial response. Science. 311:1770-1773.

58. Beck, M.A. 2007. Selenium and vitamin E status: impact on viral pathogenicity. J. Nutr. 137:1338-1340. 